\title{
Complexity of Deep Inference via Atomic Flows
}

\author{
Anupam Das *
}

\begin{abstract}
We consider the fragment of deep inference free of compression mechanisms and compare its proof complexity to other systems, utilising 'atomic flows' to examine size of proofs. Results include a simulation of Resolution and dag-like cut-free Gentzen, as well as a separation from bounded-depth Frege.
\end{abstract}

\section{Introduction}

Deep inference differs from other proof formalisms by allowing derivations themselves to be composed by logical connectives. There has recently been a lot of activity in the proof complexity of deep inference [2], most notably that a cut-free system, $\mathrm{KS}^{+}$, quasipolynomially simulates Frege systems [12] [3]. It is conjectured that this can be improved to a polynomial simulation, so finding lower bounds for $\mathrm{KS}^{+}$is probably as hard as finding one for Frege, which has escaped proof complexity theorists for years.

However this quasipolynomial simulation relies crucially on the presence of daglike behaviour, manifested in deep inference by a particular rule, cocontraction: $\frac{A}{A \wedge A}$. Without it we have a minimal complete system closed under deep inference, KS. This system is free of compression mechanisms, in that a proof of a conjunction can be 'partitioned' into proofs of each conjunct, unlike proofs that are dag-like or contain cut.

It is conjectured that $\mathrm{KS}$ is unable to polynomially simulate $\mathrm{KS}^{+}$[2], raising the question of exactly where it fits in the hierarchy of proof systems.

In this paper we focus on upper bounds and simulations to demonstrate the relative strength of KS. Our arguments employ atomic flows [10], diagrams that track structural changes in a proof but forget logical information, to show that cocontraction, and certain other steps, can be sometimes eliminated from a proof in polynomial time. A comprehensive introduction to atomic flows can be found in [11].

Our main result is a polynomial simulation of dag-like cut-free Gentzen systems (dag-Gen ${ }^{-}$) in KS, improving on the simulation of the tree-like system in [2]. This also places KS in the gap between dag- $\mathrm{Gen}^{-}$and a variation augmented with elimination rules $\left(\mathrm{Gen}^{\star}\right)$, shown in [7] to simulate $\mathrm{KS}^{+}$, thereby quasipolynomially simulating Frege by the aforementioned result. This is discussed further in conclusion 7.2

Fig. 11 summarises our results, and full proofs of results can be found in [8]

\section{Deep Inference}

We work in propositional logic over the basis $\{\bar{\cdot}, \wedge, \vee\}$ with formulae in negation normal form. Syntactic equivalence of formulae is denoted $\equiv$.

Definition 1 (Rules and Systems). An inference rule is a binary relation on formulae decidable in polynomial time, and a system is a set of rules. We define the rules we use

* a.das abath.ac.uk. Department of Computer Science, University of Bath. 


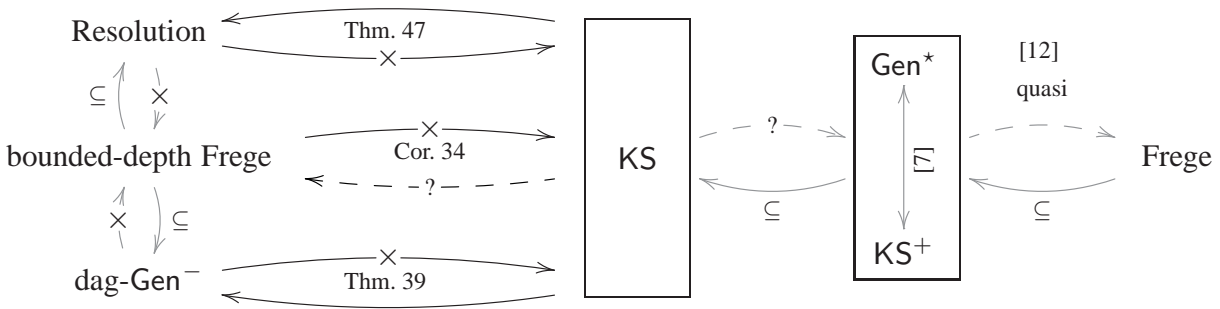

Fig. 1: Relative complexity of systems after results in this paper.

below, and the systems $\mathrm{KS}=\{\mathrm{ai} \downarrow, \mathrm{aw} \downarrow, \mathrm{ac} \downarrow, \mathrm{s}, \mathrm{m}\}, \mathrm{KS}^{+}=\mathrm{KS} \cup\{\mathrm{aw} \uparrow, \mathrm{ac} \uparrow\}, \mathrm{SKS}=$ $\mathrm{KS}^{+} \cup\{$ ai $\uparrow$ and $\overline{\mathrm{KS}}=\{\mathrm{ai} \uparrow, \mathrm{aw} \uparrow, \mathrm{ac} \uparrow, \mathrm{s}, \mathrm{m}\}$.

We also have a logical rule $=$ which allows us to apply laws of associativity, commutativity and basic equations with units [2].

Atomic structural rules

$$
\begin{array}{cccc}
\text { ai } \downarrow \frac{\mathrm{t}}{a \vee \bar{a}} & \text { aw } \downarrow \frac{\mathrm{f}}{a} & \mathrm{ac} \downarrow \frac{a \vee a}{a} & \mathrm{~s} \frac{A \wedge[B \vee C]}{(A \wedge B) \vee C} \\
\text { identity } & \text { weakening } & \text { contraction } & \text { switch } \\
\mathrm{a} \uparrow \frac{a \wedge \bar{a}}{\mathrm{f}} & \text { aw } \uparrow \frac{a}{\mathrm{t}} & \mathrm{ac} \uparrow \frac{a}{a \wedge a} & \mathrm{~m} \frac{(A \wedge B) \vee(C \wedge D)}{[A \vee C] \wedge[B \vee D]} \\
\text { cut } & \text { coweakening } & \text { cocontraction } & \text { medial }
\end{array}
$$

Linear logical rules

Definition 2 (Proofs and Derivations). We define derivations, and premiss and conclusion functions ( $\mathrm{pr}, \mathrm{cn}$ resp.), inductively. Every formula $A$ is a derivation with $\operatorname{pr}(A) \equiv$ $A \equiv \operatorname{cn}(A)$. For derivations $\Phi, \Psi:$ if $\star \in\{\wedge, \vee\}$ then $\Phi \star \Psi$ is a derivation with premiss $\operatorname{pr}(\Phi) \star \operatorname{pr}(\Psi)$ and conclusion $\operatorname{cn}(\Phi) \star \operatorname{cn}(\Psi)$; if $\rho \frac{\operatorname{cn}(\Phi)}{\operatorname{pr}(\Psi)}$ is an instance of a rule $\rho, \rho \frac{\Phi}{\Psi}$ is a derivation with premiss $\operatorname{pr}(\Phi)$ and conclusion $\mathrm{cn}(\Psi)$.

If $\operatorname{pr}(\Phi) \equiv \mathrm{t}$ then we call $\Phi$ a proof. If $\Phi$ is a derivation in a system $\mathcal{S}$ with premiss

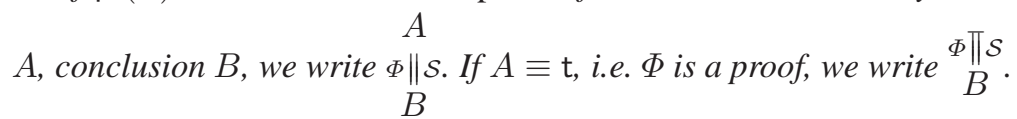

Proposition 3 ([1] $)$. Each rule $\rho$ below is derivable in $\{\mathrm{s}, \mathrm{m}, \mathrm{a} \rho\}$ :

$$
i \downarrow \frac{\mathrm{t}}{A \vee \bar{A}} \quad \mathrm{w} \downarrow \frac{\mathrm{f}}{A} \quad \mathrm{c} \downarrow \frac{A \vee A}{A} \quad \mathrm{i \uparrow} \frac{A \wedge \bar{A}}{\mathrm{f}} \quad \mathrm{w} \uparrow \frac{A}{\mathrm{t}} \quad \mathrm{c} \uparrow \frac{A}{A \wedge A}
$$

We will use the above 'generic' rules as abbreviations for their derivations. 


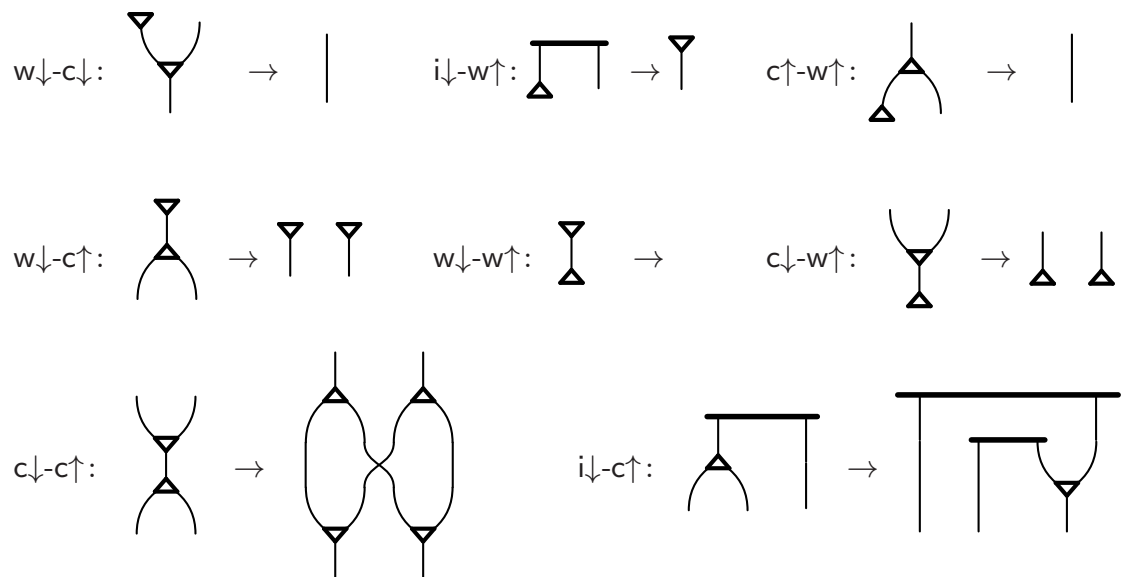

Fig. 2: Local rewriting rules for the system norm.

Definition 4 (Complexity). We define the size $|\Phi|$ of a derivation $\Phi$ to be the number of atom occurrences in $\Phi$. A system $\mathcal{S}$ p-simulates a system $\mathcal{T}$ if each $\mathcal{T}$-proof can be polynomially transformed into an $\mathcal{S}$-proof of the same conclusion.

\section{Atomic Flows}

Definition 5 (Atomic Flows). For an SKS derivation $\Phi$ we define its atomic flow, $f l(\Phi)$, to be the diagram obtained by tracing the path of each atom through the derivation, designating structural rules by the following corresponding nodes:

$$
\begin{aligned}
& \mathrm{ai \downarrow} \frac{\mathrm{t}}{a \vee \bar{a}} \mapsto \prod_{\operatorname{aw} \downarrow \frac{\mathrm{f}}{a}} \mapsto Y \quad \operatorname{ac} \downarrow \frac{a \vee a}{a} \mapsto Y \\
& \mathrm{ai \uparrow} \frac{a \wedge \bar{a}}{\mathrm{f}} \mapsto \mathrm{L} \quad \operatorname{aw} \uparrow \frac{a}{\mathrm{t}} \mapsto \Delta \quad \mathrm{\Delta} \quad \mathrm{ac} \uparrow \frac{a}{a \wedge a} \mapsto
\end{aligned}
$$

We consider flows as graphs embedded in the plane with the six types of nodes above. Note that edges may be pending at either end.

We define the size of a flow $\phi$, denoted $|\phi|$, to be its number of edges.

Definition 6. We define a rewriting system norm on flows in Fig. 2

Proposition 7 ([10]). norm is terminating and confluent.

Notation 8 If a flow $\psi$ is the normal form of a flow $\phi$ under a terminating, confluent rewriting system $\mathrm{r}$, then we write $\phi \underset{\mathrm{r}}{\rightarrow} \psi$.

Definition 9. If $\mathcal{R}$ is a relation on atomic flows we say that $\mathcal{R}$ lifts polynomially to SKS if, whenever $(f l(\Phi), \psi) \in \mathcal{R}$, we can construct a derivation $\Psi$ in time polynomial in $|\Phi|+|\psi|$ with the same premiss and conclusion as $\Phi$ and atomic flow $\psi$. 
Theorem 10 ([10]). $\underset{\text { norm }}{\longrightarrow}$ lifts polynomially to SKS.

Corollary 11. If $\phi$ is the flow of a $\mathrm{KS}^{+}$proof, $\phi \underset{\mathrm{norm}}{\longrightarrow} \psi$ then $\psi$ is the flow of a $\mathrm{KS}$ proof.

Example 12. In Fig. 3 we give a derivation, its flow and a reduction under norm.

We consider atoms to be positive or negative, under some valid assignment of polarity. We use the terms 'upwards' and 'downwards' with regard to derivations and flows, interpreted in the natural way, independently from the notion of direction defined below.

Definition 13 (Paths). To each edge we assign a direction: downwards if the atom associated with it is positive, upwards if it is negative.

We define a path in a flow to be a directed path between pending edges.

Example 14. We give the following flow and all its paths:

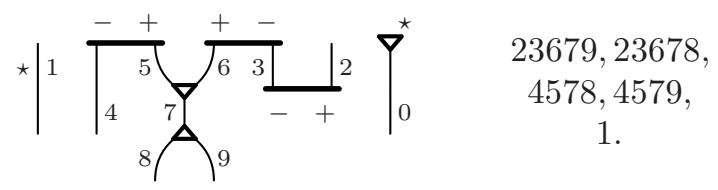

where,+- indicate the polarity of the atom associated with an edge, under some assignment, and $\star$ indicates that either polarity may be correctly assigned.

Notice that the number of paths is invariant under valid assignments of polarity.

The following results allow us to estimate the size of the normal form of a flow, under norm, without actually constructing it.

Observation 15 Reducing under norm conserves the number of paths in a flow.

Recall that, in a proof, there are no assumptions, and so the flow of a proof can have no edge with upper end pending; it must be attached to an identity or weakening node.

Let $\#(\rho, \phi)$ be the number of $\rho$-nodes in a flow $\phi$, and $\ulcorner\phi\urcorner$ be its number of paths.

Observation 16 If $\phi$ is the flow of a $\mathrm{KS}$ proof, then $\ulcorner\phi\urcorner=\#(\mathrm{ai} \downarrow, \phi)$.

Theorem 17. If $\phi$ is the flow of a $\mathrm{KS}^{+}$proof, $\phi \underset{\text { norm }}{\longrightarrow} \psi$, then $|\psi|=O(|\phi|+\ulcorner\phi\urcorner)$.

Proof. Decompose $\psi$ into its identity fragment $\psi_{1}$ and weakening fragment $\psi_{2}$. Note that each rule involving $\mathrm{w} \downarrow$ or $\mathrm{w} \uparrow$ reduces the size of the flow, so $\left|\psi_{2}\right| \leq|\phi|$.

Notice that $\left|\psi_{1}\right|=2 \cdot \#\left(\operatorname{ai} \downarrow, \psi_{1}\right)+\#\left(\operatorname{ac} \downarrow, \psi_{1}\right)$. However, clearly, the number of contractions cannot outnumber the number of edges emanating from identity steps, so we have $\left|\psi_{1}\right| \leq 4 \cdot \#\left(\right.$ ai $\left.\downarrow, \psi_{1}\right)$. By Obs. 16 we then have $\left|\psi_{1}\right| \leq 4 \cdot\ulcorner\psi\urcorner$, and by Obs.15 that $\left|\psi_{1}\right| \leq 4 \cdot\ulcorner\phi\urcorner$, whence $|\psi|=\left|\psi_{1}\right|+\left|\psi_{2}\right| \leq|\phi|+4 \cdot\ulcorner\phi\urcorner$.

Remark 18. The main contributor to an increase of flow size reducing under norm is the rule $c \downarrow-c \uparrow$. It can sometimes cause an exponential blowup [10].

The following proposition estimates the increase in size caused by $\mathrm{c} \downarrow-\mathrm{c} \uparrow$.

Proposition 19. If in every directed path of a flow $\phi$ there are at most $k$ alternations of ac $\uparrow$ and $\mathrm{ac} \downarrow$ nodes then $\ulcorner\phi\urcorner=|\phi|^{O(k)}$. 

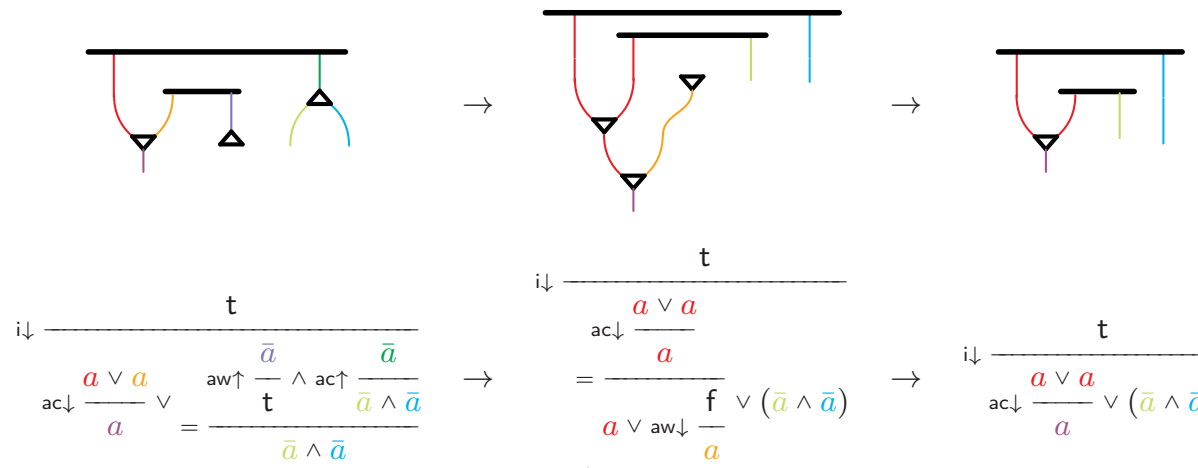

$$
=\frac{\mathrm{t}}{\mathrm{i} \downarrow} \frac{a \vee \frac{a}{a}}{a \vee \operatorname{aw} \downarrow \frac{\mathrm{f}}{a}} \vee(\bar{a} \wedge \bar{a}) \quad \rightarrow \quad i \downarrow \frac{\mathrm{t}}{\mathrm{ac} \downarrow \frac{a \vee a}{a} \vee(\bar{a} \wedge \bar{a})}
$$

Fig. 3: An example of a derivation, its flow and a reduction under norm.

\section{Truth Tables and Tableaux}

Bruscoli and Guglielmi have proved that tree-like cut-free Gentzen cannot p-simulate $\mathrm{KS}$, by way of the Statman tautologies [2]. We offer a new proof here, via truth tables.

Observation 20 A truth table proof for a formula $A$ has size $|A| \cdot 2^{\# A}$, where \#A is the number of distinct propositional variables in $A$.

Lemma 21. $\mathrm{KS}^{+}$p-simulates truth tables.

Proof. Let $\tau$ be a tautology. For each partial assignment $\mathcal{A}$, defined on just those atoms appearing in $\tau$ construct a derivation $\Phi_{\mathcal{A}}(\tau)$ by structural induction on $\tau$ :

$$
\Phi_{\mathcal{A}}(a) \equiv a \quad, \quad \Phi_{\mathcal{A}}(A \wedge B) \equiv \Phi_{\mathcal{A}}(A) \wedge \Phi_{\mathcal{A}}(B) \quad, \quad \Phi_{\mathcal{A}}(A \vee B) \equiv \frac{\Phi_{\mathcal{A}}(B)}{{ } \downarrow \frac{\mathrm{f}}{A} \vee B}
$$

where, in the last case, when $\tau$ is a disjunction, choose a disjunct $B$ that is true under $\mathcal{A}$. It is clear that each $\Phi_{\mathcal{A}}(\tau)$ has conclusion $\tau$ and premiss a conjunction of literals; moreover this conjunction of literals is satisfied by $\mathcal{A}$.

Let $\gamma_{\mathcal{A}}$ be the conjunction of all literals satisfied by $\mathcal{A}$ such that each literal appears at most once. Then there is a derivation of the form: $\gamma_{\mathcal{A}} \|$ aw $\left.\uparrow, \mathrm{ac} \uparrow\right\}$.

$$
\operatorname{pr}\left(\Phi_{\mathcal{A}}(\tau)\right)
$$

By distributivity, derived on the left, we can construct a proof $\Psi$ of $\bigvee_{\mathcal{A}} \gamma_{\mathcal{A}}$ in $\{\mathrm{ai} \downarrow, \mathrm{ac} \uparrow, \mathrm{s}, \mathrm{m}\}$, and then apply contractions to obtain the proof, on the right:

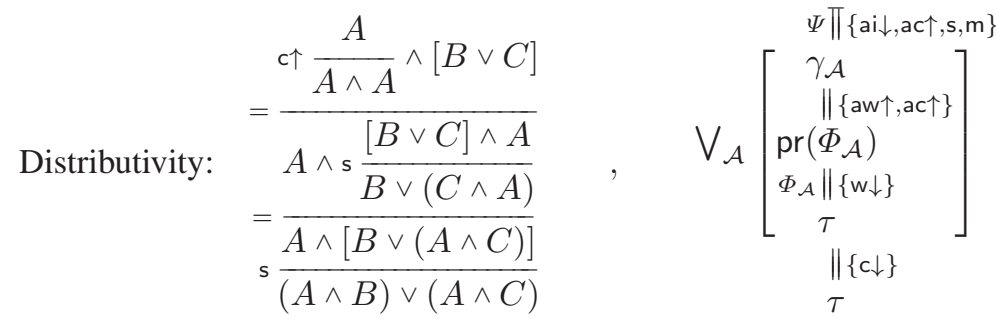


Theorem 22. KS p-simulates truth tables.

Proof. In the above proofs all $c \uparrow$ steps are above all $c \downarrow$ steps, so by Prop. 19 the number of paths is polynomial in the size of the flow. The result follows by Thms. 17 and 10

Notation 23 Let tree/dag-Gen ${ }^{-}$denote cut-free Gentzen with tree/dag proofs resp.

Proposition 24 ([[6]). Tree-Gen ${ }^{-}$cannot $p$-simulate truth tables.

Corollary 25. Tree-Gen ${ }^{-}$cannot p-simulate $\mathrm{KS}$.

Proof. Immediate from Prop. 24 and Thm.22.

\section{Separations via the Functional Pigeonhole Principle}

We show Gen ${ }^{-}$, Resolution and bounded-depth Frege systems cannot p-simulate KS, by reducing under norm Jeřábek's $\mathrm{KS}^{+}$proofs of the functional pigeonhole principle.

Conversely we give a simulation of Resolution, and some extensions, in KS.

\subsection{Polynomial-Size Proofs of the Functional Pigeonhole Principle}

The functional pigeonhole principle is a class of propositional tautologies asserting that there is no injective function from a set of size $n+1$ to a set of size $n$.

Definition 26 (Functional Pigeonhole Principle).

$$
\operatorname{FPHP}_{n} \equiv \bigvee_{i=0}^{n} \bigwedge_{j=1}^{n} \bar{a}_{i j} \vee \bigvee_{i=0}^{n} \bigvee_{j=1}^{n-1} \bigvee_{j^{\prime}=j+1}^{n}\left(a_{i j} \wedge a_{i j^{\prime}}\right) \vee \bigvee_{j=1}^{n} \bigvee_{i=0}^{n-1} \bigvee_{i^{\prime}=i+1}^{n}\left(a_{i j} \wedge a_{i^{\prime} j}\right)
$$

Theorem 27 ([15][14]). Bounded-depth Frege has only exponential-size proofs of $\mathrm{FPHP}_{n}$.

Corollary 28. Resolution and $\mathrm{Gen}^{-}$have only exponential-size proofs of $\mathrm{FPHP}_{n}$.

Theorem 29 ([4]). There are polynomial-size Frege proofs of $\mathrm{FPHP}_{n}$.

Proposition $30([2])$. SKS is polynomially equivalent to Frege systems.

Lemma 31. Every SKS proof $\Phi$ of a formula A can be polynomially transformed to a $\mathrm{KS}$ proof of $A \vee \bigvee_{i}\left(a_{i} \wedge \bar{a}_{i}\right)$, where $a_{i}$ are the distinct propositional variables in $A$.

Lemma 32 ([12]). There are polynomial-size proofs $\Theta_{n}$ in $\mathrm{KS}^{+}$of $\mathrm{FPHP}_{n}$.

Proof (Jeřábek). By Thm. 29. Prop. 30 and Lemma 31 we can build KS proofs $\Phi_{n}$ of $\mathrm{FPHP}_{n} \vee \bigvee_{i, j}\left(a_{i j} \wedge \bar{a}_{i j}\right)$ that have size polynomial in $n$. For each atom $a_{s t}$ we construct a derivation $\Psi_{n}^{a_{s t}}$ in $\mathrm{KS}^{+} \backslash\{\mathrm{ac} \downarrow\}$ from $a_{s t} \wedge \bar{a}_{s t}$ to $\mathrm{FPHP}_{n}$, on the left below, and apply contractions to obtain the proof, on the right:

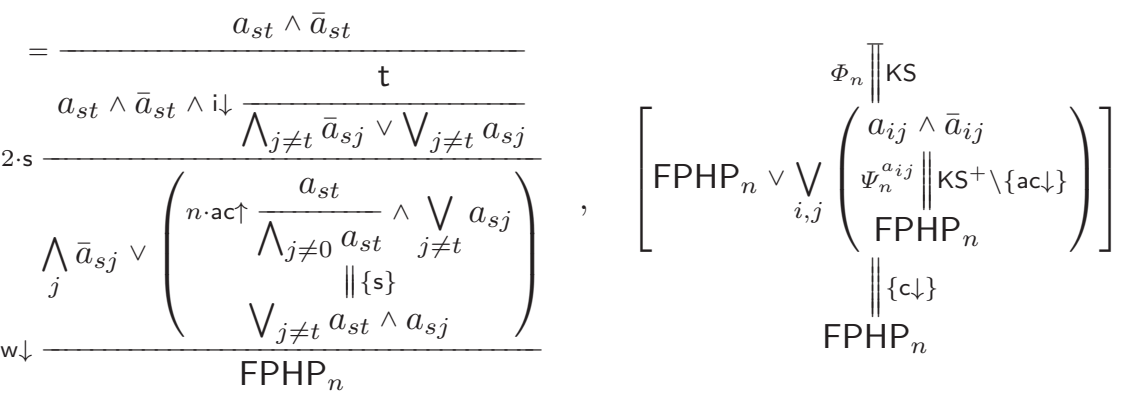


Theorem 33. There are polynomial-size proofs in $\mathrm{KS}$ of $\mathrm{FPHP}_{n}$.

Proof. In $\Theta_{n}$ above, there are 2 alternations between $c \downarrow$ and $c \uparrow$ nodes, so $\left\ulcorner f l\left(\Theta_{n}\right)\right\urcorner=$ $\left|f l\left(\Theta_{n}\right)\right|^{O(2)}$ by Prop. 19. The result follows by Thms. 17 and 10 .

Corollary 34. Gen ${ }^{-}$, Resolution, bounded-depth Frege cannot p-simulate KS.

Proof. Immediate from Thm.27 Cor.28 and Thm. 33.

\subsection{A Polynomial Simulation of Resolution and Some Extensions}

We give a p-simulation of resolution systems in KS, first noticed in [9].

Definition 35. We define Resolution by the following CNF rewriting rules:

$\operatorname{RES}=\left\{\operatorname{res} \frac{A \wedge[B \vee a] \wedge[\bar{a} \vee C]}{A \wedge[B \vee C]}\right.$, ac $\downarrow \frac{A \wedge[B \vee a \vee a]}{A \wedge[B \vee a]}$, dag $\frac{A \wedge B}{A \wedge B \wedge B}$, aw $\left.\downarrow \frac{A \wedge B}{A \wedge[B \vee b]}\right\}$ modulo associativity and commutativity. A RES refutation is a derivation $\|$ RES.

$f$

Proposition 36. Define $\mathrm{w} \downarrow-\mathrm{i} \uparrow: \underline{I} \rightarrow \downarrow$ and $\mathrm{w}=\{\mathrm{w} \downarrow-\mathrm{c} \uparrow, \mathrm{w} \downarrow-\mathrm{w} \uparrow, \mathrm{w} \downarrow-\mathrm{i} \uparrow, \mathrm{w} \downarrow-\mathrm{c} \downarrow\}$.

Then $\mathrm{w}$ is terminating, confluent and $\underset{\mathrm{w}}{\rightarrow}$ lifts polynomially to SKS. [10]

Lemma 37. RES refutations can be polynomially transformed to ones in $\overline{\mathrm{KS}}$.

Proof. We derive a generalisation res' of res on the left, and eliminate ac $\downarrow$-steps by the translation on the right. Finally, aw $\downarrow$ steps are eliminated by Prop. 36 .

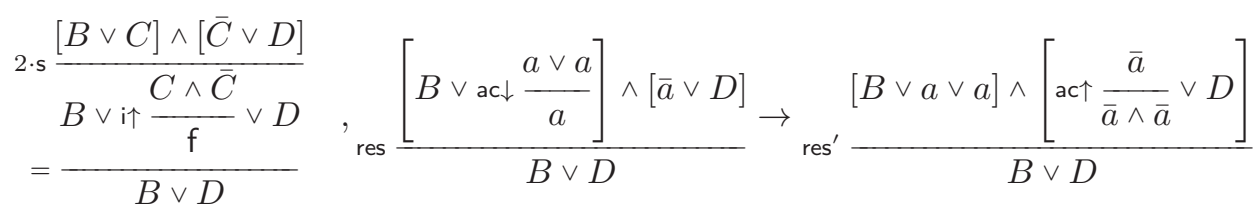

Lemma 38. We can transform a $\overline{\mathrm{KS}}$ refutation of $\bar{A}$ in linear time to a $\mathrm{KS}$ proof of $A$.

Proof (Sketch). 'Flip' the refutation upside-down, replace every formula with its negation and every 'up' rule with its corresponding 'down' rule.

Theorem 39. KS p-simulates Resolution systems.

Proof. Immediate from Lemmata 38 and 37

Finally, the simulation above can be extended to some basic extensions of Resolution, introduced by Krajíček [13], where literals are replaced by conjunctions of literals.

Definition 40. RES $(f)$ consists of the rules of RES, with atomic variables varying over conjunctions of literals, and the rule $\wedge \frac{A \wedge\left[B \vee \bigwedge_{i} a_{i}\right] \wedge\left[\bigwedge_{j} b_{j} \vee C\right]}{A \wedge\left[B \vee\left(\bigwedge_{i} a_{i} \wedge \bigwedge_{j} b_{j}\right) \vee C\right]}$.

Additionally, in a derivation $\Phi$, no conjunction of literals may be larger than $f(|\Phi|)$.

Proposition 41. KS $p$-simulates $\operatorname{RES}(f)$ for any function $f$.

Proof. $\wedge$ is derivable in $\{s\}$, and the old rules can be dealt with as before. 


\section{A Simulation of Dag-Like Cut-Free Gentzen}

We consider Gen in its one-sided variation, e.g. GS1p in [16], and identify sequents with the disjunction of their formulae, as an abuse of notation.

We now give a translation of dag-like cut-free Gentzen proofs to $\mathrm{KS}^{+}$, and then KS. Naively we could just apply a generic cocontraction to simulate each dag-step, duplicating the entire sequent, but this may lead to an exponential blowup reducing under norm by Rmk. 18 .

Instead we notice that, when two branches of a dag step are brought together by a $\wedge$ step, we only need to cocontract the formulae which are common ancestors to the conjuncts of the $\wedge$ step. For example:

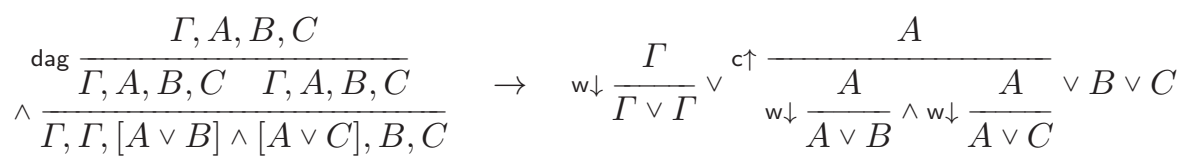

When there are other rules between the dag and $\wedge$ steps, we can translate them into deep steps, inside the conjunction $[A \vee B] \wedge[A \vee C]$ above, for example.

Definition 42. For a sequent $\Gamma$ and formula $A$ occurring in a $\mathrm{Gen}^{-}$derivation $\pi$, let $\operatorname{Anc}_{\Gamma}(A)$ denote the set of ancestors of $A$ occurring in $\Gamma$.

Definition 43. A contraction loop in a flow $\phi$ is a $(\mathrm{c} \uparrow, \mathrm{c} \downarrow)$ pair of nodes $\left(\nu_{1}, \nu_{2}\right)$ in $\phi$, with $\nu_{1}$ above $\nu_{2}$, where there are two (or more) disjoint paths between $\nu_{1}$ and $\nu_{2}$.

Lemma 44. There is a polynomial transformation $T$ from dag-Gen ${ }^{-}$derivations to $\mathrm{KS}^{+}$satisfying the following properties:

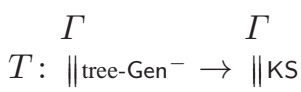

$$
\begin{aligned}
& \Delta \quad \Delta
\end{aligned}
$$

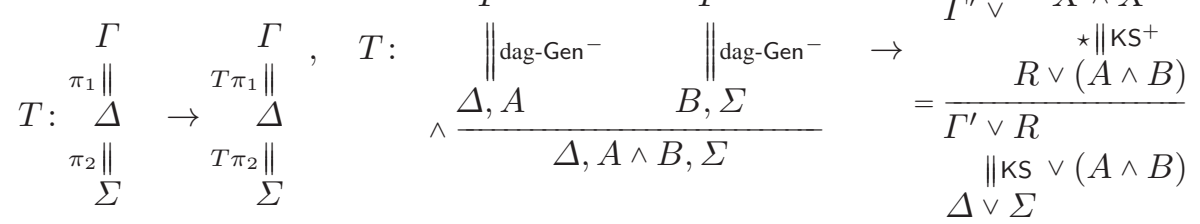

where $X=\operatorname{Anc}_{\Gamma}(A) \cap \operatorname{Anc}_{\Gamma}(B)$ and $\Gamma^{\prime}=\Gamma \backslash X, R$ is some formula, and there are no contraction loops in $\star$.

Observation 45 If $\pi$ is a dag-Gen ${ }^{-}$derivation then, by the subformula property and the properties in Lemma 43 there are no contraction loops in $f l(T \pi)$.

Lemma 46. If there are no contraction loops in a flow $\phi$ then $\ulcorner\phi\urcorner$ is polynomial in $|\phi|$.

Proof. Define c: $\bigcap_{>} \rightarrow \mathrm{c}$ is terminating, confluent and if $\phi \underset{\mathrm{c}}{\rightarrow} \psi$ then $|\phi|=|\psi|$ and $\ulcorner\phi\urcorner \leq\ulcorner\psi\urcorner$. If $\phi$ has no contraction loops then, in $\psi$, all $\mathrm{c} \downarrow$-nodes are above all c个-nodes. So $\ulcorner\phi\urcorner \leq\ulcorner\psi\urcorner=|\psi|^{O(3)}=|\phi|^{O(3)}$ by Prop.19. 
Theorem 47. KS p-simulates dag-like cut-free Gentzen systems.

Proof. Immediate from Obs.45, Lemmata46, 43 and Thms.17,10

\section{Conclusions}

We have seen that KS is a surprisingly powerful system, despite lacking any mechanism to compress proofs. As well as the simulations of Resolution and dag-like cut-free Gentzen, it cannot be p-simulated by bounded-depth Frege, one of the strongest 'weak' systems, and also has polynomial-size proofs of the functional pigeonhole principle.

\subsection{Atomic Flows as a Tool for Complexity Analysis}

Atomic flows are a useful tool to analyse and manipulate derivations; often we can avoid the possibly exponential blowup in eliminating cocontractions. Further work could investigate whether we can always avoid this blowup, via a local or global flow reduction.

\subsection{Dag-Like Cut-Free Gentzen Systems and Variations}

Results in [7] show that the addition of elimination rules (below) to dag-Gen ${ }^{-}$result in a system $\mathrm{Gen}^{\star}$ that is p-equivalent to $\mathrm{KS}^{+}$, and so quasipolynomially simulates Frege.

$$
\vee \text { - } \operatorname{Elim} \frac{\Gamma, A \vee B}{\Gamma, A, B} \quad, \quad \wedge-\operatorname{Elim}-\mathrm{L} \frac{\Gamma, A \wedge B}{\Gamma, A} \quad, \quad \wedge \text {-Elim-R } \frac{\Gamma, A \wedge B}{\Gamma, B}
$$

On the other hand we showed that, without these modifications, $\mathrm{Gen}^{-}$cannot even psimulate KS, and in fact that KS fits neatly between these two variations:

$$
\text { dag-Gen }^{-}<\mathrm{KS} \leqq \text { ? } \mathrm{KS}^{+}=\mathrm{Gen}^{\star} \leqq \text { Frege }
$$

The restriction on proofs caused by the subformula property seems to be critical; it would be interesting to investigate its effects on proof complexity in general.

We regard KS to be an uncompressed system: every proof of a conjunction $A \wedge B$ can be partitioned into a proof of $A$ and a proof of $B$, with no sharing between them, by substituting $\mathrm{t}$ for one of the conjuncts and reducing every line in the proof by $=$.

Consequently, for any dag-Gen ${ }^{-}$proof of a conjunction $A \wedge B$ there are $\mathrm{KS}$ proofs of $A$ and $B$ whose sizes sum to the size of the initial proof, for some notion of size globally accurate up to a polynomial. We thus argue that the sharing effect of dagness in cut-free Gentzen systems serves solely to do some of the work of deep inference, but not all of it due to the strict separation between the two systems.

We notice that the separation of $\mathrm{KS}_{\text {and }}$ tree-Gen ${ }^{-}$in [2] is in fact just a special case of Thm.47, since dag-like cut-free Gentzen has polynomial-size proofs of the Statman tautologies [5].

\subsection{Stronger Systems}

We showed that bounded-depth Frege cannot p-simulate KS but did not consider the other direction. We conjecture that they are incomparable, due to the dissimilar ways they are defined. Similar questions persist for other 'stronger' systems, e.g. Cutting Planes, although ongoing research suggests we may be able to obtain a separation of KS from Cutting Planes. 
Acknowledgements The author would like to thank Alessio Guglielmi and Tom Gundersen for all their time and effort discussing this paper with me, and the anonymous referees for their useful comments.

\section{References}

1. K. Brünnler and A. F. Tiu. A local system for classical logic. Number WV-01-02, 2001. http://iccl.tu-dresden.de/ kai/LocalClassicalLogic-tr.pdf

2. P. Bruscoli and A. Guglielmi. On the proof complexity of deep inference. ACM Transactions on Computational Logic, 10(2):1-34, 2009. Article 14. http://cs.bath.ac.uk/ag/p/PrComplDI.pdf

3. P. Bruscoli, A. Guglielmi, T. Gundersen, and M. Parigot. Quasipolynomial normalisation in deep inference via atomic flows and threshold formulae. Submitted. http://cs.bath.ac.uk/ag/p/QuasiPolNormDI.pdf, 2009.

4. S. R. Buss. Polynomial size proofs of the propositional pigeonhole principle. Journal of Symbolic Logic, 52(4):916-927, 1987.

5. P. Clote and E. Kranakis. Boolean Functions and Computation Models. Springer-Verlag, 2002.

6. M. D'Agostino. Are tableaux an improvement on truth-tables? Journal of Logic, Language and Information, 1:235-252, 1992. 10.1007/BF00156916.

7. A. Das. On the proof complexity of cut-free bounded deep inference. Tableaux '11., 2011.

8. A. Das. Complexity of deep inference via atomic flows. 2012. http://www.anupamdas.com/items/RelComp/RelComp.pdf

9. A. Guglielmi. Resolution in the calculus of structures. http://cs.bath.ac.uk/ag/p/AG10.pdf, 2003.

10. A. Guglielmi and T. Gundersen. Normalisation control in deep inference via atomic flows II. http://cs.bath.ac.uk/ag/p/NormContrDIAtFl2.pdf 2008.

11. T. Gundersen. A General View of Normalisation Through Atomic Flows. PhD thesis, University of Bath, 2009.

12. E. Jeřábek. Proof complexity of the cut-free calculus of structures. volume 19, pages 323 339, 2009. http://www.math.cas.cz/ jerabek/papers/cos.pdf.

13. J. Krajíček. On the weak pigeonhole principle. 2001.

14. J. Krajíček, P. Pudlk, and A. Woods. An exponential lower bound to the size of bounded depth frege proofs of the pigeonhole principle. Random Structures \& Algorithms, 7(1).

15. T. Pitassi, P. Beame, and R. Impagliazzo. Exponential lower bounds for the pigeonhole principle. Computational Complexity, 3:97-140, 1993. 10.1007/BF01200117.

16. A. Troelstra and H. Schwichtenberg. Basic Proof Theory, volume 43 of Cambridge Tracts in Theoretical Computer Science. Cambridge University Press, 1996. 